\title{
Prevalence of oral mucosal lesions among fishermen of Kutch coast, Gujarat, India
}

\author{
Santhosh Vediyera Chandroth ${ }^{1}$, Harish Kumar Vadakkedath Venugopal ${ }^{1}$, Saji Puthenveetil ${ }^{2}$, \\ Arpitha Jayaram ${ }^{3}$, Jacob Mathews ${ }^{4}$, Nandan Suresh ${ }^{5}$, Abdulaziz Abdullah Al Kheraif ${ }^{6}$, \\ Ravikumar Ramakrishnaiah ${ }^{6}$, Darshan Devang Divakar ${ }^{6}$, Kailash Asawa $^{7}$, Aniruddh Tak ${ }^{8}$, Mridula Tak ${ }^{7}$ \\ ${ }^{1}$ KMCT Dental College, Kerala University of Health and Allied Science, Kerala, India \\ ${ }^{2}$ Department of Prosthodontics, Pariyaram Dental College, Kerala, India \\ ${ }^{3}$ Department of Orthodontics, AIMST University, Malaysia \\ ${ }^{4}$ Department of Periodontics, Indian Dental School and Hospital, Mauritius \\ ${ }^{5}$ Department of Pedodontics and Preventive Dentistry, KVG Dental College and Hospital Karnataka, India \\ ${ }^{6}$ Dental Biomaterials Research Chair, Dental Health Department, College of Applied Medical Sciences, \\ King Saud University, Kingdom of Saudi Arabia \\ ${ }^{7}$ Department of Public Health Dentistry, Pacific Dental College and Hospital, Udaipur, Rajasthan, India \\ ${ }^{8}$ Department of Oral Pathology and Microbiology, Pacific Dental College and Hospital, Udaipur, Rajasthan, India
}

\begin{abstract}
Background: The aim of the study was to assess the prevalence of oral mucosal lesions among fishermen population in the coastal region of Kutch, Gujarat, India.

Materials and methods: A descriptive cross-sectional survey was conducted to assess the prevalence of oral mucosal lesions among the 979 fishermen of Kutch District, Gujarat, India. The proforma included information on demographic details, habits (tobacco and alcohol), oral hygiene practices and presence and location of oral mucosal lesions according to World Health Organisation, 2013. Chi-square test was used for comparisons. Confidence level and p-value were set at $95 \%$ and $5 \%$, respectively.

Results: The majority of study population consumed tobacco and alcohol (88.1\%) in some or the other form and used chewsticks (42.9\%) for cleaning their teeth. Amongst all, 30.03\% of the study subjects suffered from oral mucosal lesions. Leukoplakia (13.8\%) was found to be the most prevalent lesion. The most affected sites were lips and vermillion border. The prevalence of oral mucosal lesions was found to be significantly associated with age, gender, oral hygiene practices and adverse habits.

Conclusions: Oral mucosal lesions were prevalent among $30.03 \%$ of the study population. More detailed studies probing this issue should be conducted and efforts should be directed towards primordial prevention of the conditions.
\end{abstract}

(Int Marit Health 2014; 65, 4: 192-198)

Key words: prevalence, oral mucosal lesions, Kutch

\section{INTRODUCTION}

Oral cavity is prone for a myriad of changes with advancing age as well as a result of the environmental and life style related factors [1]. From a histological and embryological point of view, the mucosa of the oral-cavity is similar to the skin, but it is subjected to a more complex and inconstant environment. This complex nature modifies the pattern of disease presentation in mouth [2]. Oral lesions can interfere with daily social activities in involved patients through impacts on mastication, swallowing and speech and symptoms like xerostomia, halitosis or dysesthesia [3].

Oral mucosal lesions can occur as a result of infections, local trauma or irritation, systemic diseases and excessive consumption of tobacco, betel quid and alcohol [1]. Fishing is an occupation, associated with irregular diet, stress, alcoholism, tobacco consumption and pernicious habits. 
Fishermen have lower socio-economic status and their illiteracy adds to their poor oral hygiene, which may influence general and oral health. Fishermen have prolonged hours of continuous work, which are found to be correlated with high cigarette and alcohol consumption. Diet lacks fruits and vegetables and meals are eaten at very erratic intervals $[4,5]$. Moreover, fishermen are prone to excess ultraviolet radiation due to constant exposure to sun. Few studies have been conducted exploring the oral health of fishermen populations, and a thorough literature search did not reveal any studies focusing on oral mucosal lesions.

Hence, owing to dearth of literature and to gather baseline data for planning of oral health promotion programs, the present study was conducted with an objective to assess the prevalence of oral mucosal lesions among fishermen population in the coastal region of Kutch, Gujarat, India.

\section{MATERIALS AND METHODS}

\section{STUDY DESIGN, POPULATION AND DURATION}

A descriptive cross-sectional survey was conducted to assess the prevalence of oral mucosal lesions among the fishermen community of Kutch District, Gujarat, India, from January 2014 to March 2014.

\section{OFFICIAL PERMISSION AND ETHICAL CLEARANCE}

The study protocol was reviewed by the Ethical Committee of Pacific Dental College and Hospital and the ethical clearance was granted. An official permission was obtained from the Chairman of Fisherfolk community of Kutch District, Gujarat, India.

\section{INFORMED CONSENT}

After explaining the purpose and details of the study, a written informed consent was obtained from all the subjects who were willing to participate. Illiterate subjects were explained about the details of the study by the investigator and their thumb impressions were taken on the consent form.

\section{TRAINING AND CALIBRATION}

All the examinations were carried out by a single qualified examiner. Training sessions and calibration of examiner were conducted by the 2 oral medicine consultants until an acceptable level of consistency in diagnosis was reached. The intra-examiner reliability was assessed using Kappa statistics and was found to be $95 \%$.

\section{INCLUSION AND EXCLUSION CRITERIA}

The inclusion criteria include: (1) Those who were willing to participate and (2) Patients aged 18 years and above.

The exclusion criteria include: (1) Those who were not willing to participate and (2) Patients who were severely ill.

\section{PILOT SURVEY}

A pilot study was carried out among 50 fishermen subjects to determine the feasibility and practicability of the study and the time required for examination of each subject. It helped to know the practical difficulties while conducting the survey. It took around $10 \mathrm{~min}$ to assess each subject. The prevalence of oral mucosal lesions was found to be $29 \%$.

\section{SAMPLE SIZE CALCULATION}

Depending on the prevalence of oral mucosal lesions obtained (29\%), 95\% confidence level and 10\% allowable error, the minimum sample size was determined to be 979 .

\section{SAMPLING DESIGN}

Multistage random sampling was employed to select the study population. Kutch coast is divided into 4 zones from which 1 zone (Anjar, Mundra) was randomly selected.

The principal unit of administration in India is the district under a Collector. Most districts are divided into 2 or more subdivisions. Each subdivision is again divided into Tehsils. Each Tehsil comprises of several villages.

From the selected zone, a tehsil (Mundra) was randomly selected. From the selected tehsil, 2 villages (Bhadreshwar and Luni) were randomly selected. Samples were selected using probability proportional sampling from each village. Subjects were selected starting from the reference point (north east corner of selected villages) employing simple random sampling technique until the minimum sample size was achieved.

\section{PROFORMA DETAILS AND CLINICAL EXAMINATION}

The proforma included information on demographic details, habits (tobacco and alcohol), oral hygiene practices and presence and location of oral mucosal lesions [6]. A portable dental chair and light were used to facilitate outreach examinations and field work.

\section{STATISTICAL ANALYSIS}

The recorded data was compiled and entered in a spreadsheet computer program (Microsoft Excel 2007) and then exported to data editor page of SPSS version 11.5 (SPSS Inc., Chicago, Illinois, USA). Chi-square test was used for comparisons. Confidence level and $p$-value were set at $95 \%$ and $5 \%$, respectively.

\section{RESULTS}

Table 1 presents the distribution of study population by age, gender and adverse habits. The majority of the study population were males (57.4\%). Amongst all, only $7.2 \%$ subjects were 68 years of age and above. The majority of 
Table 1. Distribution of study population according to age, gender and adverse habits

\begin{tabular}{|c|c|c|c|c|c|c|c|c|}
\hline \multirow[t]{2}{*}{ Variables } & \multicolumn{6}{|c|}{ Adverse habits } & \multirow[t]{2}{*}{ Total } & \multirow[t]{2}{*}{$\mathbf{P}$} \\
\hline & None & $\begin{array}{l}\text { Smoking } \\
\text { tobacco }\end{array}$ & $\begin{array}{l}\text { Smokeless } \\
\text { tobacco }\end{array}$ & $\begin{array}{l}\text { Smoking + } \\
\text { + smokeless } \\
\text { tobacco }\end{array}$ & Alcohol & $\begin{array}{l}\text { Tobacco + } \\
+ \text { alcohol }\end{array}$ & & \\
\hline Age [years] & & & & & & & & $0.03 *$ \\
\hline $18-27$ & 17 (8.9\%) & 40 (21.1\%) & 36 (18.9\%) & 27 (14.2\%) & 41 (21.6\%) & 29 (15.3\%) & $190(19.4 \%)$ & \\
\hline $28-37$ & $14(8.3 \%)$ & 38 (22.6\%) & $38(22.6 \%)$ & 24 (14.3\%) & 33 (19.6\%) & $21(12.5 \%)$ & $168(17.2 \%)$ & \\
\hline $38-47$ & $18(9.4 \%)$ & 37 (19.3\%) & $48(25 \%)$ & 31 (16.1\%) & 27 (14.1\%) & 31 (16.1\%) & $192(19.6 \%)$ & \\
\hline $48-57$ & 14 (7.1\%) & 44 (22.4\%) & 42 (21.4\%) & 36 (18.4\%) & 35 (17.9\%) & 25 (12.8\%) & $196(20 \%)$ & \\
\hline $58-67$ & 31 (19.01\%) & 27 (16.6\%) & 41 (25.2\%) & 25 (15.3\%) & 13 (7.9\%) & 26 (15.9\%) & $163(16.6 \%)$ & \\
\hline 68 and above & 23 (32.9\%) & $10(14.3 \%)$ & 9 (12.9\%) & 15 (21.4\%) & 2 (2.9\%) & $11(15.7 \%)$ & 70 (7.2\%) & \\
\hline Gender & & & & & & & & $0.02 *$ \\
\hline Male & 48 (8.5\%) & 101 (17.9\%) & $113(20.1 \%)$ & 99 (17.6\%) & $111(19.8 \%)$ & $90(16.01 \%)$ & $562(57.4 \%)$ & \\
\hline Female & 69 (16.5\%) & 95 (22.8\%) & $101(24.2 \%)$ & 59 (14.1\%) & $40(9.6 \%)$ & $53(12.7 \%)$ & 417 (42.6\%) & \\
\hline Total & 117 (11.9\%) & $196(20.02 \%)$ & $214(21.9 \%)$ & $158(16.1 \%)$ & $151(15.4 \%)$ & $143(14.6 \%)$ & $979(100 \%)$ & \\
\hline
\end{tabular}

Test applied: Chi-square test, ${ }^{*} p<0.05$ (statistically significant)

Table 2. Distribution of study population according to age, gender and oral hygiene practices

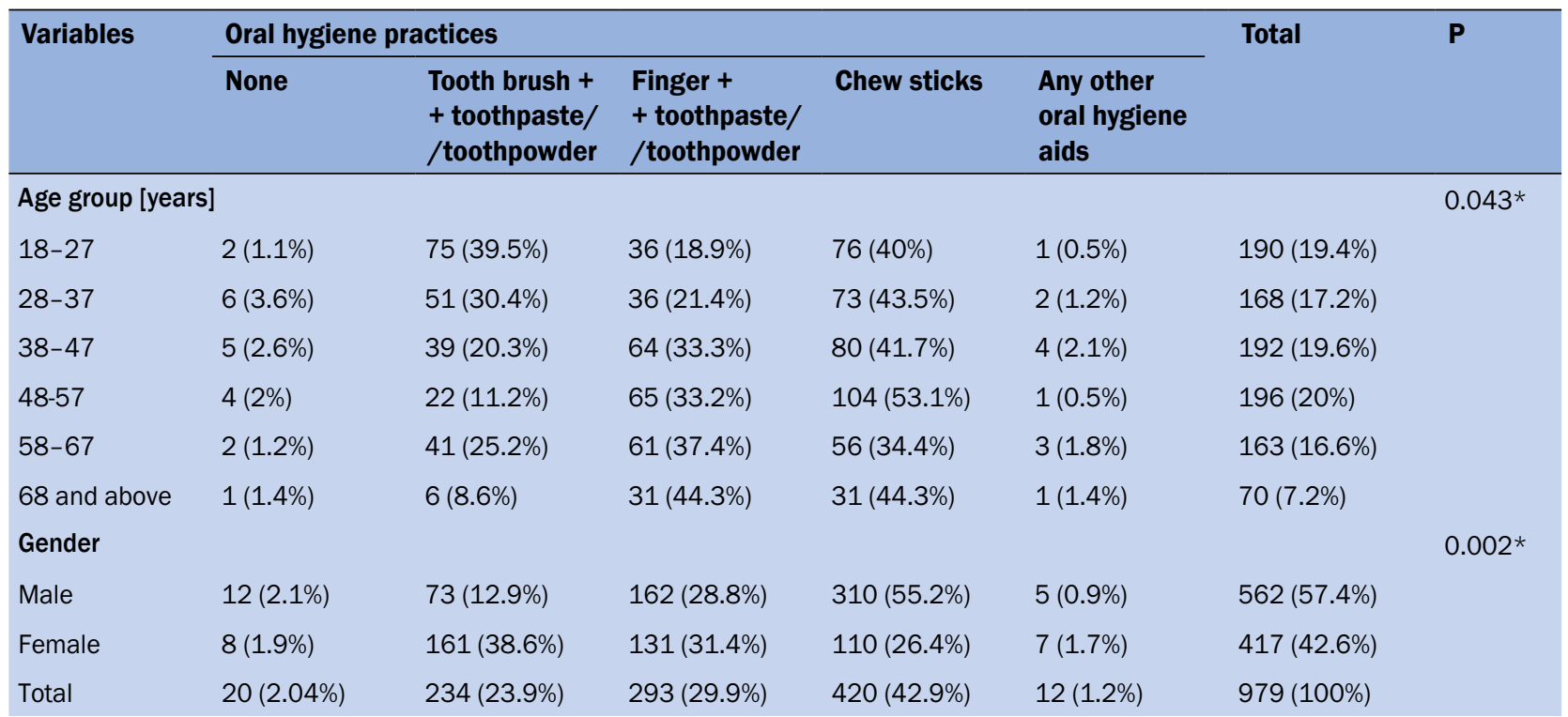

Test applied: Chi-square test, ${ }^{\star} \mathrm{p}<0.05$ (statistically significant)

them (88.1\%) consumed tobacco and alcohol in some or the other form. Smokeless tobacco (21.9\%) was the most prevalent tobacco habit followed by smoking tobacco (20.02\%). Significantly ( $p=0.03$ ) fewer subjects above 58 years of age had adverse habits.

Table 2 elicits oral hygiene practices of study population by age and gender. The most of the study population used chewsticks (42.9\%) for cleaning their teeth followed by finger and toothpaste/toothpowder (29.9\%). The usage of toothbrush and tooth paste decreased significantly $(p=0.043)$ with increasing age. Significantly $(p=0.002)$ greater proportion of females (38.6\%) used toothbrush and toothpaste than males (57.4\%).

Tables 3 and 4 depict the prevalence of oral mucosal lesions according to several independent variables. Amongst all, $30.03 \%$ of the study subjects suffered from oral mucosal lesions. Leukoplakia (13.8\%) was found to be the most prevalent lesion followed by ulceration (7.2\%) and other lesions (4.8\%) like oral submucous fibrosis and cheilitis. Malignant tumour, lichen planus and acute necrotising 
Table 3. Prevalence of oral mucosal lesions according to age and gender

\begin{tabular}{|c|c|c|c|c|c|c|c|c|c|c|c|}
\hline \multirow[t]{2}{*}{ Variables } & \multicolumn{10}{|c|}{ Oral mucosal lesions } & \multirow[t]{2}{*}{$\mathbf{P}$} \\
\hline & $\begin{array}{l}\text { No abnor- } \\
\text { mal con- } \\
\text { dition }\end{array}$ & $\begin{array}{l}\text { Mali- } \\
\text { gnant } \\
\text { tumour }\end{array}$ & $\begin{array}{l}\text { Leuko- } \\
\text { plakia }\end{array}$ & $\begin{array}{l}\text { Lichen } \\
\text { planus }\end{array}$ & $\begin{array}{l}\text { Ulcera- } \\
\text { tion }\end{array}$ & $\begin{array}{l}\text { Acute ne- } \\
\text { crotizing } \\
\text { gingivitis }\end{array}$ & $\begin{array}{l}\text { Candi- } \\
\text { diasis }\end{array}$ & Abscess & Others & $\begin{array}{l}\text { Total } \\
\text { lesions }\end{array}$ & \\
\hline Age group & & & & & & & & & & & $0.032 *$ \\
\hline $\begin{array}{l}18-27 \\
(n=190)\end{array}$ & $\begin{array}{l}158 \\
(83.2 \%)\end{array}$ & $\begin{array}{l}0 \\
(0 \%)\end{array}$ & $\begin{array}{l}15 \\
(7.9 \%)\end{array}$ & $\begin{array}{l}1 \\
(0.5 \%)\end{array}$ & $\begin{array}{l}10 \\
(5.3 \%)\end{array}$ & $\begin{array}{l}0 \\
(0 \%)\end{array}$ & $\begin{array}{l}0 \\
(0 \%)\end{array}$ & $\begin{array}{l}2 \\
(1.1 \%)\end{array}$ & $\begin{array}{l}4 \\
(2.1 \%)\end{array}$ & $\begin{array}{l}32 \\
(16.8 \%)\end{array}$ & \\
\hline $\begin{array}{l}28-37 \\
(n=168)\end{array}$ & $\begin{array}{l}113 \\
(67.3 \%)\end{array}$ & $\begin{array}{l}0 \\
(0 \%)\end{array}$ & $\begin{array}{l}20 \\
(11.9 \%)\end{array}$ & $\begin{array}{l}0 \\
(0 \%)\end{array}$ & $\begin{array}{l}29 \\
(17.3 \%)\end{array}$ & $\begin{array}{l}0 \\
(0 \%)\end{array}$ & $\begin{array}{l}0 \\
(0 \%)\end{array}$ & $\begin{array}{l}0 \\
(0 \%)\end{array}$ & $\begin{array}{l}6 \\
(3.6 \%)\end{array}$ & $\begin{array}{l}55 \\
(32.7 \%)\end{array}$ & \\
\hline $\begin{array}{l}38-47 \\
(n=192)\end{array}$ & $\begin{array}{l}132 \\
(68.8 \%)\end{array}$ & $\begin{array}{l}2 \\
(1.04 \%)\end{array}$ & $\begin{array}{l}25 \\
(13.02 \%)\end{array}$ & $\begin{array}{l}2 \\
(1.04 \%)\end{array}$ & $\begin{array}{l}15 \\
(7.8 \%)\end{array}$ & $\begin{array}{l}0 \\
(0 \%)\end{array}$ & $\begin{array}{l}5 \\
(2.6 \%)\end{array}$ & $\begin{array}{l}1 \\
(0.5 \%)\end{array}$ & $\begin{array}{l}10 \\
(5.2 \%)\end{array}$ & $\begin{array}{l}60 \\
(31.3 \%)\end{array}$ & \\
\hline $\begin{array}{l}48-57 \\
(n=196)\end{array}$ & $\begin{array}{l}124 \\
(63.3 \%)\end{array}$ & $\begin{array}{l}3 \\
(1.5 \%)\end{array}$ & $\begin{array}{l}35 \\
(17.9 \%)\end{array}$ & $\begin{array}{l}3 \\
(1.5 \%)\end{array}$ & $\begin{array}{l}10 \\
(5.1 \%)\end{array}$ & $\begin{array}{l}0 \\
(0 \%)\end{array}$ & $\begin{array}{l}0 \\
(0 \%)\end{array}$ & $\begin{array}{l}6 \\
(3.1 \%)\end{array}$ & $\begin{array}{l}15 \\
(7.7 \%)\end{array}$ & $\begin{array}{l}72 \\
(36.7 \%)\end{array}$ & \\
\hline $\begin{array}{l}58-67 \\
(n=163)\end{array}$ & $\begin{array}{l}105 \\
(64.4 \%)\end{array}$ & $\begin{array}{l}1 \\
(0.6 \%)\end{array}$ & $\begin{array}{l}32 \\
(19.6 \%)\end{array}$ & $\begin{array}{l}1 \\
(0.6 \%)\end{array}$ & $\begin{array}{l}4 \\
(2.5 \%)\end{array}$ & $\begin{array}{l}2 \\
(1.2 \%)\end{array}$ & $\begin{array}{l}3 \\
(1.8 \%)\end{array}$ & $\begin{array}{l}5 \\
\text { (3.1\%) }\end{array}$ & $\begin{array}{l}10 \\
(6.1 \%)\end{array}$ & $\begin{array}{l}58 \\
(35.6 \%)\end{array}$ & \\
\hline $\begin{array}{l}68 \text { and } \\
\text { above } \\
(n=70)\end{array}$ & $\begin{array}{l}53 \\
(75.7 \%)\end{array}$ & $\begin{array}{l}0 \\
(0 \%)\end{array}$ & $\begin{array}{l}8 \\
(11.4 \%)\end{array}$ & $\begin{array}{l}0 \\
(0 \%)\end{array}$ & $\begin{array}{l}2 \\
(2.9 \%)\end{array}$ & $\begin{array}{l}0 \\
(0 \%)\end{array}$ & $\begin{array}{l}2 \\
(2.9 \%)\end{array}$ & $\begin{array}{l}3 \\
(4.3 \%)\end{array}$ & $\begin{array}{l}2 \\
(2.9 \%)\end{array}$ & $\begin{array}{l}17 \\
(24.3 \%)\end{array}$ & \\
\hline Gender & & & & & & & & & & & $0.001 *$ \\
\hline $\begin{array}{l}\text { Male } \\
(n=562)\end{array}$ & $\begin{array}{l}380 \\
(67.6 \%)\end{array}$ & $\begin{array}{l}4 \\
(0.7 \%)\end{array}$ & $\begin{array}{l}82 \\
(14.6 \%)\end{array}$ & $\begin{array}{l}5 \\
(0.9 \%)\end{array}$ & $\begin{array}{l}48 \\
(8.5 \%)\end{array}$ & $\begin{array}{l}2 \\
(0.4 \%)\end{array}$ & $\begin{array}{l}7 \\
(1.2 \%)\end{array}$ & $\begin{array}{l}8 \\
(1.4 \%)\end{array}$ & $\begin{array}{l}26 \\
(4.6 \%)\end{array}$ & $\begin{array}{l}182 \\
(32.4 \%)\end{array}$ & \\
\hline $\begin{array}{l}\text { Female } \\
(n=417)\end{array}$ & $\begin{array}{l}305 \\
\text { (73.1\%) }\end{array}$ & $\begin{array}{l}2 \\
(0.5 \%)\end{array}$ & $\begin{array}{l}53 \\
(12.7 \%)\end{array}$ & $\begin{array}{l}2 \\
(0.5 \%)\end{array}$ & $\begin{array}{l}22 \\
(5.3 \%)\end{array}$ & $\begin{array}{l}0 \\
(0 \%)\end{array}$ & $\begin{array}{l}3 \\
(0.7 \%)\end{array}$ & $\begin{array}{l}9 \\
(2.2 \%)\end{array}$ & $\begin{array}{l}21 \\
(5 \%)\end{array}$ & $\begin{array}{l}112 \\
(26.7 \%)\end{array}$ & \\
\hline
\end{tabular}

Test applied: Chi-square test, ${ }^{*} p<0.05$ (statistically significant)

gingivitis affected only a very small number of subjects. Older age groups (28-67 years) revealed significantly greater proportion of subjects with lesions $(p=0.032)$ than younger (18-27 years) age groups. Gender distribution showed that males (32.4\%) were more affected than females (26.7\%) ( $p=0.001$ ). Amongst all, $74.1 \%$ of those who consumed both tobacco and alcohol and around half of the subjects who consumed both smoking and smokeless tobacco suffered from oral mucosal lesions. The group with no adverse habit also revealed $8.5 \%$ subjects who suffered from oral mucosal lesions. The comparison based on adverse habits showed statistically significant difference $(p=0.022)$. Subjects with no oral hygiene practices $(75 \%)$ showed significantly $(p=0.01$ ) highest proportion of subjects with lesions followed by those using chewsticks (39.3\%).

Table 5 revealed that the majority of participants presented with lesions on lips (53.4\%) followed by vermillion border (23.5\%) and commissures (10.9\%).

\section{DISCUSSION}

The present study aimed to assess the prevalence of oral mucosal lesions among fishermen population in the coastal region of Kutch, Gujarat, India. A total of 979 fishermen participated in the study.

Literature search revealed that the present study is a pioneer study by itself, hence results of the study are compared with results of studies assessing information about general populations.

The present study population was in the age range of 18-68 years and above with the majority subjects in the age range of $18-48$ years and only a small proportion belonging to the age group of 49-68 years. Pertaining to gender, an approximately equal distribution was evident (males $57.4 \%$, females $42.6 \%$ ). The majority (97.9\%) of the study population had some or the other adverse habits. The prevalence obtained was much higher than among the general population of Chennai, India where the overall prevalence of smoking, smokeless tobacco and alcohol consumption were $15.02 \%, 6.99 \%$ and $8.78 \%$, respectively [7]. The prevalence of adverse habits in the present study was also greater than among the general population of Vidisha where only half of the study subjects consumed tobacco/ /alcohol [8]. This higher proportion of fishermen with adverse habits may be due to their laborious occupation and less awareness of hazards owing to their low educational background. In the present study, smokeless tobacco, found to be the most prevalent adverse habit, was used by $21.9 \%$ of subjects, which is a little less than the proportion of subjects consuming pan masala (Pan masala consists of areca nuts [betel nuts], catechu, lime cardamom, spices, and unspecified flavouring agents, etc. with tobacco; locally known as gutkha or without tobacco [plain]) in the coastal 
Table 4. Prevalence of oral mucosal lesions according to adverse habits and oral hygiene practices

\begin{tabular}{|c|c|c|c|c|c|c|c|c|c|c|c|}
\hline \multirow[t]{2}{*}{ Variables } & \multicolumn{10}{|c|}{ Oral mucosal lesions } & \multirow[t]{2}{*}{$\mathbf{P}$} \\
\hline & $\begin{array}{l}\text { No abnor- } \\
\text { mal con- } \\
\text { dition }\end{array}$ & $\begin{array}{l}\text { Mali- } \\
\text { gnant } \\
\text { tumour }\end{array}$ & $\begin{array}{l}\text { Leuko- } \\
\text { plakia }\end{array}$ & $\begin{array}{l}\text { Lichen } \\
\text { planus }\end{array}$ & $\begin{array}{l}\text { Ulcera- } \\
\text { tion }\end{array}$ & $\begin{array}{l}\text { Acute ne- } \\
\text { crotizing } \\
\text { gingivitis }\end{array}$ & $\begin{array}{l}\text { Candi- } \\
\text { diasis }\end{array}$ & $\begin{array}{l}\text { Ab- } \\
\text { scess }\end{array}$ & Others & $\begin{array}{l}\text { Total } \\
\text { lesions }\end{array}$ & \\
\hline \multicolumn{11}{|c|}{ Adverse habits } & \multirow[t]{7}{*}{$0.022 *$} \\
\hline $\begin{array}{l}\text { None } \\
(n=117)\end{array}$ & $\begin{array}{l}107 \\
(91.5 \%)\end{array}$ & $\begin{array}{l}0 \\
(0 \%)\end{array}$ & $\begin{array}{l}1 \\
(0.9 \%)\end{array}$ & $\begin{array}{l}0 \\
(0 \%)\end{array}$ & $\begin{array}{l}5 \\
(4.3 \%)\end{array}$ & $\begin{array}{l}0 \\
(0 \%)\end{array}$ & $\begin{array}{l}1 \\
(0.9 \%)\end{array}$ & $\begin{array}{l}0 \\
(0 \%)\end{array}$ & $\begin{array}{l}3 \\
(2.7 \%)\end{array}$ & $\begin{array}{l}10 \\
(8.5 \%)\end{array}$ & \\
\hline $\begin{array}{l}\text { Smoking } \\
\text { tobacco } \\
(n=196)\end{array}$ & $\begin{array}{l}162 \\
(82.7 \%)\end{array}$ & $\begin{array}{l}2 \\
(1 \%)\end{array}$ & $\begin{array}{l}7 \\
(3.6 \%)\end{array}$ & $\begin{array}{l}1 \\
(0.5 \%)\end{array}$ & $\begin{array}{l}16 \\
(8.2 \%)\end{array}$ & $\begin{array}{l}0 \\
(0 \%)\end{array}$ & $\begin{array}{l}2 \\
(1 \%)\end{array}$ & $\begin{array}{l}2 \\
(1 \%)\end{array}$ & $\begin{array}{l}4 \\
(2 \%)\end{array}$ & $\begin{array}{l}34 \\
(17.3 \%)\end{array}$ & \\
\hline $\begin{array}{l}\text { Smokeless } \\
\text { tobacco } \\
(n=214)\end{array}$ & $\begin{array}{l}176 \\
(82.2 \%)\end{array}$ & $\begin{array}{l}0 \\
(0 \%)\end{array}$ & $\begin{array}{l}6 \\
(2.8 \%)\end{array}$ & $\begin{array}{l}0 \\
(0 \%)\end{array}$ & $\begin{array}{l}12 \\
(5.6 \%)\end{array}$ & $\begin{array}{l}1 \\
(0.5 \%)\end{array}$ & $\begin{array}{l}1 \\
(0.5 \%)\end{array}$ & $\begin{array}{l}2 \\
(0.9 \%)\end{array}$ & $\begin{array}{l}16 \\
(7.5 \%)\end{array}$ & $\begin{array}{l}38 \\
(17.8 \%)\end{array}$ & \\
\hline $\begin{array}{l}\text { Smoking + } \\
+ \text { smokeless } \\
\text { tobacco } \\
(n=158)\end{array}$ & $\begin{array}{l}76 \\
(48.1 \%)\end{array}$ & $\begin{array}{l}1 \\
(0.6 \%)\end{array}$ & $\begin{array}{l}51 \\
(32.3 \%)\end{array}$ & $\begin{array}{l}3 \\
(1.9 \%)\end{array}$ & $\begin{array}{l}21 \\
(13.3 \%)\end{array}$ & $\begin{array}{l}1 \\
(0.6 \%)\end{array}$ & $\begin{array}{l}3 \\
(1.9 \%)\end{array}$ & $\begin{array}{l}1 \\
(0.6 \%)\end{array}$ & $\begin{array}{l}1 \\
(0.6 \%)\end{array}$ & $\begin{array}{l}82 \\
(51.9 \%)\end{array}$ & \\
\hline $\begin{array}{l}\text { Alcohol } \\
(n=151)\end{array}$ & $\begin{array}{l}127 \\
(84.1 \%)\end{array}$ & $\begin{array}{l}0 \\
(0 \%)\end{array}$ & $\begin{array}{l}8 \\
(5.3 \%)\end{array}$ & $\begin{array}{l}1 \\
(0.7 \%)\end{array}$ & $\begin{array}{l}6 \\
(3.9 \%)\end{array}$ & $\begin{array}{l}0 \\
(0 \%)\end{array}$ & $\begin{array}{l}1 \\
(0.7 \%)\end{array}$ & $\begin{array}{l}4 \\
(2.6 \%)\end{array}$ & $\begin{array}{l}4 \\
(2.6 \%)\end{array}$ & $\begin{array}{l}24 \\
(15.9 \%)\end{array}$ & \\
\hline $\begin{array}{l}\text { Tobacco + } \\
+ \text { alcohol } \\
(n=143)\end{array}$ & $\begin{array}{l}37 \\
(25.9 \%)\end{array}$ & $\begin{array}{l}3 \\
(2.1 \%)\end{array}$ & $\begin{array}{l}62 \\
(43.4 \%)\end{array}$ & $\begin{array}{l}2 \\
(1.4 \%)\end{array}$ & $\begin{array}{l}10 \\
(6.9 \%)\end{array}$ & $\begin{array}{l}0 \\
(0 \%)\end{array}$ & $\begin{array}{l}2 \\
(1.4 \%)\end{array}$ & $\begin{array}{l}8 \\
(5.6 \%)\end{array}$ & $\begin{array}{l}19 \\
(13.3 \%)\end{array}$ & $\begin{array}{l}106 \\
(74.1 \%)\end{array}$ & \\
\hline \multicolumn{11}{|c|}{ Oral hygiene practices } & \multirow[t]{7}{*}{$0.01 *$} \\
\hline $\begin{array}{l}\text { None } \\
(n=20)\end{array}$ & $\begin{array}{l}5 \\
(25 \%)\end{array}$ & $\begin{array}{l}0 \\
(0 \%)\end{array}$ & $\begin{array}{l}0 \\
(0 \%)\end{array}$ & $\begin{array}{l}2 \\
(10 \%)\end{array}$ & $\begin{array}{l}2 \\
(10 \%)\end{array}$ & $\begin{array}{l}2 \\
(10 \%)\end{array}$ & $\begin{array}{l}4 \\
(20 \%)\end{array}$ & $\begin{array}{l}0 \\
(0 \%)\end{array}$ & $\begin{array}{l}5 \\
(25 \%)\end{array}$ & $\begin{array}{l}15 \\
(75 \%)\end{array}$ & \\
\hline $\begin{array}{l}\text { Tooth brush + } \\
+ \text { toothpaste/ } \\
\text { /toothpowder } \\
(\mathrm{n}=234)\end{array}$ & $\begin{array}{l}202 \\
(86.3 \%)\end{array}$ & $\begin{array}{l}0 \\
(0 \%)\end{array}$ & $\begin{array}{l}9 \\
(3.8 \%)\end{array}$ & $\begin{array}{l}0 \\
(0 \%)\end{array}$ & $\begin{array}{l}15 \\
(6.4 \%)\end{array}$ & $\begin{array}{l}0 \\
(0 \%)\end{array}$ & $\begin{array}{l}0 \\
(0 \%)\end{array}$ & $\begin{array}{l}0 \\
(0 \%)\end{array}$ & $\begin{array}{l}8 \\
(3.4 \%)\end{array}$ & $\begin{array}{l}32 \\
(13.7 \%)\end{array}$ & \\
\hline $\begin{array}{l}\text { Finger + } \\
+ \text { toothpaste/ } \\
\text { /toothpowder } \\
(n=293)\end{array}$ & $\begin{array}{l}223 \\
(76.1 \%)\end{array}$ & $\begin{array}{l}3 \\
(1.02 \%)\end{array}$ & $\begin{array}{l}21 \\
(7.2 \%)\end{array}$ & $\begin{array}{l}1 \\
(0.3 \%)\end{array}$ & $\begin{array}{l}22 \\
(7.5 \%)\end{array}$ & $\begin{array}{l}0 \\
(0 \%)\end{array}$ & $\begin{array}{l}3 \\
(1.02 \%)\end{array}$ & $\begin{array}{l}7 \\
(2.4 \%)\end{array}$ & $\begin{array}{l}13 \\
(4.4 \%)\end{array}$ & $\begin{array}{l}70 \\
(23.9 \%)\end{array}$ & \\
\hline $\begin{array}{l}\text { Chew sticks } \\
(n=420)\end{array}$ & $\begin{array}{l}255 \\
(60.7 \%)\end{array}$ & $\begin{array}{l}1 \\
(0.2 \%)\end{array}$ & $\begin{array}{l}103 \\
(24.5 \%)\end{array}$ & $\begin{array}{l}3 \\
(0.7 \%)\end{array}$ & $\begin{array}{l}24 \\
(5.7 \%)\end{array}$ & $\begin{array}{l}0 \\
(0 \%)\end{array}$ & $\begin{array}{l}3 \\
(0.7 \%)\end{array}$ & $\begin{array}{l}10 \\
(2.4 \%)\end{array}$ & $\begin{array}{l}21 \\
(5 \%)\end{array}$ & $\begin{array}{l}165 \\
(39.3 \%)\end{array}$ & \\
\hline $\begin{array}{l}\text { Any other } \\
\text { oral hygiene } \\
\text { aids } \\
(n=12)\end{array}$ & $\begin{array}{l}0 \\
(0 \%)\end{array}$ & $\begin{array}{l}2 \\
(16.7 \%)\end{array}$ & $\begin{array}{l}2 \\
(16.7 \%)\end{array}$ & $\begin{array}{l}1 \\
(8.3 \%)\end{array}$ & $\begin{array}{l}7 \\
(58.3 \%)\end{array}$ & $\begin{array}{l}0 \\
(0 \%)\end{array}$ & $\begin{array}{l}0 \\
(0 \%)\end{array}$ & $\begin{array}{l}0 \\
(0 \%)\end{array}$ & $\begin{array}{l}0 \\
(0 \%)\end{array}$ & $\begin{array}{l}12 \\
(100 \%)\end{array}$ & \\
\hline $\begin{array}{l}\text { Total } \\
(\mathrm{n}=979)\end{array}$ & $\begin{array}{l}685 \\
(69.9 \%)\end{array}$ & $\begin{array}{l}6 \\
(0.6 \%)\end{array}$ & $\begin{array}{l}135 \\
(13.8 \%)\end{array}$ & $\begin{array}{l}7 \\
(0.7 \%)\end{array}$ & $\begin{array}{l}70 \\
(7.2 \%)\end{array}$ & $\begin{array}{l}2 \\
(0.2 \%)\end{array}$ & $\begin{array}{l}10 \\
(1.02 \%)\end{array}$ & $\begin{array}{l}17 \\
(1.7 \%)\end{array}$ & $\begin{array}{l}47 \\
(4.8 \%)\end{array}$ & $\begin{array}{l}294 \\
(30.03 \%)\end{array}$ & \\
\hline
\end{tabular}

Test applied: Chi-square test, ${ }^{\star} \mathrm{p}<0.05$ (statistically significant)

Table 5. Distribution of study population by site of the lesion

\begin{tabular}{ll}
\hline Site of the lesion & Number (\%) \\
\hline Vermillion border & $69(23.5 \%)$ \\
Commissures & $32(10.9 \%)$ \\
Lips & $157(53.4 \%)$ \\
Sulci & $4(1.4 \%)$ \\
Buccal mucosa & $19(6.5 \%)$ \\
Floor of the mouth & $6(2 \%)$ \\
Tongue & $0(0 \%)$ \\
Hard and/or soft palate & $2(0.7 \%)$ \\
Alveolar ridge/gingiva & $5(1.7 \%)$ \\
Total & $294(100 \%)$
\end{tabular}

areas of Kerala (28.3\%) [9]. The discrepancy may be due to the difference in age groups observed in the studies. Age and gender difference in adverse habits revealed in the present study concords with the previous literature $[7,10]$. The oral hygiene habits of the study population revealed that most of them (42.9\%) used chewsticks followed by finger with toothbrush/toothpaste (29.9\%). Tooth brush and tooth paste was the mode of cleaning teeth among $23.9 \%$ of the present study population in contrast to $58 \%$ subjects from general population of Jodhpur, Rajasthan, India [11]. The preference for chewsticks and finger for cleaning teeth among present study population might be due to unaffordability of toothpaste due to lower socioeconomic status, 
lower awareness level and lower priority given to oral health. Age and gender difference in oral hygiene habits revealed in the present study are concordant with the previous literature $[12,13]$. The prevalence of oral mucosal lesions observed in the present study population was $30.03 \%$. Anecdotal prevalence of oral mucosal lesions has been reported in various populations. Greater occurrence was observed in the studies conducted at Kuwait (58.1\%) [14], Slovenia (61.6\%) [15], Philippines (61.0\%) [16], Spain (58.8\%) [17] and Thailand (83.6\%) [18]. The prevalence of oral lesions found in studies among Turin (25.1\%) [19] and Vidisha populations (8.4\%) [8] was found to be much lower than that of our study. The varied prevalence obtained in different studies may be due to differences in age groups studied and also may be due to use of different indices for recording oral mucosal lesions. Leukoplakia (13.8\%) was found to be the most prevalent lesion followed by ulceration (7.2\%) and other lesions (4.8\%) like oral submucous fibrosis and cheilitis. Moreover, it was observed that the most affected oral sites were lips (53.4\%) and vermillion border (23.5\%). The prevalence of leukoplakia in present study is much higher than that reported among South Indian population [7], Kuwaiti population [14] and South Brazilian population [20]. Leukoplakia and oral submucous fibrosis may be attributed to the presence of adverse habits in the present study. All forms of tobacco use are major risk factors for oral leukoplakia. The association is strong, consistent, reproducible, biologically plausible and shows a strong dose-response relationship. The study conducted by Piñera-Marques et al. [21] and Silva et al. [22] reported $12.8 \%$ and $43.2 \%$ fishermen with actinic lesions, respectively, which are much higher than that observed in the present study. Actinic lesions in fishermen population might be related to occupational exposure to the sun. Those who work in the open air can receive higher doses of UV radiation than those who work indoors. Lucena et al. [23] also reported high prevalence of orolabial lesions in beach workers exposed to sunlight.

The present study portrayed a greater prevalence of oral lesions among older age groups as compared to younger age groups which is in agreement with previous researches on general population groups $[14,24,25]$. Male preponderance of oral lesions depicted in present study is supported by other studies $[7,14]$. Fishermen with combination of habits (like tobacco and alcohol; smoking and smokeless tobacco) had significantly higher proportion of subjects with lesions than those on only smoking, alcohol or smokeless tobacco habits in the present study. Those with no adverse habits showed lower prevalence than other groups. The relationship between adverse habits and oral lesions has been supported by various studies $[8,14,26]$. Association of oral hygiene practices and oral mucosal lesions depicted in the present study is supported by studies conducted by Tarquinio et al. [27] and Nevalainen et al. [28] among general populations of South Brazil (2013) [27] and Finland (1997) [28], respectively.

One of the limitations of the present study was that diagnosis of lesions was done on the basis of clinical examination. Hence, more detailed studies employing investigations to confirm the diagnosis are warranted.

The benign nature of most of the lesions observed in the present study emphasizes the importance of employing conservative measures such as habit cessation, periodic re-evaluation and long-term follow-up. Efforts to increase patient awareness of the oral effects of tobacco use and to eliminate the habit are needed to improve oral and general health. Control measures should be directed towards occupational exposure to sun. Simple protection measures (covering exposed sites while working, wearing headgear or using other ways to protect the head and face) would lead to a significant reduction in new cases. Comprehensive strategies have to be developed to prevent or control oral lesions by increasing the public awareness and ways to address the problem, promote healthy oral habits and to ensure access to effective use of regular preventive and curative dental care in community based set up. The results of this study should serve as the basis for a larger, nation-wide survey of oral lesions among fishermen population.

\section{CONCLUSIONS}

The results of the present study revealed that $30.03 \%$ of the study population had oral mucosal lesions with a clear association to adverse habits (tobacco and alcohol consumption), inadequate oral hygiene practices and exposure to the sun. Initiatives directed towards prevention of disease and promotion of health should be undertaken. More comprehensive multicentre study should be conducted to get a detailed picture of the situation among the vulnerable fishermen group of India.

\section{ACKNOWLEDGEMENTS}

The authors would like to express their appreciation to the Research Centre, College of Applied Medical Sciences and the Deanship of Scientific Research at King Saud University for funding this research.

\section{REFERENCES}

1. Sridharan G. Epidemiology, control and prevention of tobacco induced oral mucosal lesions in India. Indian J Cancer 2014; 51: 80-85.

2. Babu RA, Chandrashekar P, Kumar KK et al. A study on oral mucosal lesions in 3500 patients with dermatological diseases in South India. Ann Med Health Sci Res 2014; 4 (suppl. 2): S84-S93.

3. Mansour Ghanaei F, Joukar F, Rabiei M, Dadashzadeh A, Kord Valeshabad A. Prevalence of oral mucosal lesions in an adult Iranian population. Iran Red Crescent Med J 2013; 15: $600-604$. 
4. Casson FF, Zucchero A, BoscoloBariga A et al. Work and chronic health effects among fishermen in Chioggia, Italy. G Ital Med LavErgon 1998; 20: 68-74.

5. Lawrie T, Matheson C, Ritchie L, Murphy E, Bond C. The health and lifestyle of Scottish fishermen: a need for health promotion. Health Educ Res 2004; 19: 373-379.

6. World Health Organisation. Oral health surveys. Basic methods. $5^{\text {th }}$ Ed. WHO, Geneva 2013.

7. Saraswathi TR, Ranganathan K, Shanmugam S, Sowmya R, Narasimhan PD, Gunaseelan R. Prevalence of oral lesions in relation to habits: Cross sectional study in South India. Indian J Dent Res 2006; 17: 121-125.

8. Mehrotra R, Thomas S, Nair P et al. Prevalence of oral soft tissue lesions in Vidisha. BMC Res Notes 2010; 3: 23.

9. Sandhya GI, Sunil S, Gopakumar D, Abraham B. Prevalence of pan masala use in a coastal population of Kerala. Oral Max Path J 2013; 4: 355-359.

10. Townsend J, Roderick P, Cooper J. Cigarette smoking by socioeconomic group, sex, and age: effects of price, income, and health publicity. BMJ 1994; 309: 923-927.

11. Jain N, Mitra D, Ashok KP, Dundappa J, Soni S, Ahmed S. Oral hygiene-awareness and practice among patients attending OPD at Vyas Dental College and Hospital, Jodhpur. J Indian Soc Periodontol 2012; 16: 524-528.

12. Sakthi SS, John J, Saravanan S, Kumar RP. Periodontal health status and treatment needs among building construction workers in Chennai, India. J Int Oral Health 2011; 3: 7-14.

13. Umesi-Koleoso DC, Ayanbadejo PO. Oral hygiene practices among adolescents in Surulere, Lagos state, Nigeria. Nig Q J Hosp Med 2007; 17: 112-115.

14. Ali M, Joseph B, Sundaram D. Prevalence of oral mucosal lesions in patients of the Kuwait University Dental Center. Saudi Dent J 2013; 25: 111-118.

15. Kovac-Kovacic M, Skaleric U. The prevalence of oral mucosal lesions in a population in Ljubljana. Slovenia J Oral Pathol Med 2000; 29: 331-335.

16. Cadugo MA, Chua MG, Feliciano MA, Jimenez Jr FC, Uy HG. A preliminary clinical study on the oral lesions among the Dumagats. J Philipp Dent Assoc 1998; 50: 36-42.
17. García-Pola Vallejo MJ, Martínez Díaz-Canel Al, García Martín JM, González García M. Risk factors for oral soft tissue lesions in an adult Spanish population. Community Dent Oral Epidemiol 2002; 30: 277-285.

18. Martínez Díaz-Canel Al, Garcia-Pola Vallejo MJ. Epidemiological study of oral mucosa pathology in patients of the Oviedo School of Stomatology. Med Oral 2002; 7: 4-16.

19. Pentenero M, Broccoletti R, Carbone M, Conrotto D, Gandolfo S. The prevalence of oral mucosal lesions in adults from the Turin area. Oral Dis 2008; 14: 356-366.

20. Carrard V, Haas A, Rados $P$ et al. Prevalence and risk indicators of oral mucosal lesions in an urban population from South Brazil. Oral Dis $2011 ; 17: 171-179$.

21. Piñera-Marques K, Lorenço SV, Silva LF, Sotto MN, Carneiro PC. Actinic lesions in fishermen's lower lip: clinical, cytopathological and histopathologic analysis. Clinics (Sao Paulo) 2010; 65: 363-367.

22. Silva FD, Daniel FI, Grando $\sqcup$, Calvo MC, Rath IBS, Fabro SML. Estudo da prevalência de alterações labiais em pescadores da llha de Santa Catarina. Rev Odonto Cienc. 2006; 21: 239-242.

23. Lucena EE, Costa DC, Silveira ÉJ, Lima KC. Prevalence and factors associated with orolabial lesions in beach workers. Rev Saude Publica 2012; 46: 1051-1057.

24. Al-Mobeeriek A, Al-Dosari AM. Prevalence of oral lesions among Saudi dental patients. Ann Saudi Med 2009; 29: 365-368.

25. Castellanos JL, Diaz-Guzman L. Lesions of the oral mucosa: an epidemiological study of 23785 Mexican patients. Oral Surg Oral Med Oral Pathol Oral Radiol Endod 2008; 105: 79-85.

26. Al-Attas SA, Ibrahim SS, Amer HA, DarwishZel-S, Hassan MH. Prevalence of potentially malignant oral mucosal lesions among tobacco users in Jeddah, Saudi Arabia. Asian Pac J Cancer Prev 2014; 15: 757-762.

27. Tarquinio SB, Oliveira $\sqcup$, Correa MB et al. Factors associated with prevalence of oral lesions and oral self-examination in young adults from a birth cohort in Southern Brazil. Cad Saude Publica 2013; 29: 155-164.

28. Nevalainen MJ, Närhi TO, Ainamo A. Oral mucosal lesions and oral hygiene habits in the home-living elderly. J Oral Rehabil 1997; 24: 332-337. 